\title{
Peak-To-Average Power Ratio Reduction of Ofdm Siganls
}

\author{
${ }^{1}$ Ms. Nilofer. SK, ${ }^{2} \mathrm{Mr}$.Shaik. Umar Faruq M.Tech, (Ph.d) \\ ${ }^{1}$ Student (M,Tech), ECE Department, QUBA College of engineering and Technology, Andhra Pradesh, India. \\ ${ }^{2}$ Associate Professor, ECE Department, QUBA College of engineering and Technology, Andhra Pradesh, India.
}

\begin{abstract}
Orthogonal frequency division multiplexing (OFDM) is a form of multicarrier modulation technique with high spectral efficiency and immunity to interference. Despite of many advantages the main disadvantage of OFDM is that the time domain of OFDM signal which is a sum of subcarrier sinusoids leads to high Peak-toAverage power ratio (PAPR). A simple technique used to reduce the PAPR of OFDM signals is to clip the signal to a maximum allowed value, at the cost of bit error rate (BER) degradation and out-of-band radiation. The other method is Selective mapping method (SLM) technique which is a probabilistic technique for PAPR reduction with aim of reducing the occurrence of peaks in a signal. In this project partial transmit (PTS) scheme along with the threshold technique has been simulated using Matlab and the simulation results shows that the technique performs better. To compare the results SLM and clipping methods are also simulated.

Index Terms: Bit Error Rate (BER) Degradation, Inverse Discrete Fourier Transform (IDFT), Inverse Fast Fourier Transform (IFFT), Orthogonal Frequency Division Multiplexing (OFDM)), Partial Transmit Scheme (PTS), Peak-to-Average power ratio (PAPR) and Selective Mapping Method (SLM).
\end{abstract}

\section{Introduction}

In the last years wireless communications have experienced a fast growth due to the high mobility that they allow. However wireless channels have some disadvantages like multipath fading and inter channel interference (ICI). The modulation that effectively deals with multipath fading is OFDM. As OFDM is a Multicarrier modulation technique splits the high rate data stream into $\mathrm{N}$ sub streams of lower data rate. The parallel systems divide the available bandwidth into $\mathrm{N}$ non overlapping sub channels. Each sub channel is modulated with a separate symbol and then the $\mathrm{N}$ sub channels are frequency multiplexed. The OFDM modulated signal can be expressed as

$$
\mathrm{x}(\mathrm{t})=\sum_{0}^{N-1} X_{n} \exp (2 \pi f t) \text { for }\left(0<\mathrm{t}<\mathrm{T}_{\mathrm{s}}\right)[1]
$$

Where $T_{s}$ is the duration of an OFDM symbol $1 / T_{s}$ is the distance between sub carriers(or sub channel space) in frequency domain $(\Delta \mathrm{f})$ and $\mathrm{S}_{\mathrm{n}}$ a block of $\mathrm{N}$ data complex symbols chosen from a signal constellation like QAM or PSK. Each sub carrier is located at

$$
\mathrm{f}_{\mathrm{n}}=\mathrm{nT}_{\mathrm{s}} \quad \text { for } \quad(0<\mathrm{n}<\mathrm{N}-1) \quad[2]
$$

In order to maintain the orthogonality between the OFDM symbols, the symbol duration and sub channel space must meet the condition $\mathrm{T}_{\mathrm{s}} \Delta f=1$.This way inter channel interference is eliminated but it leads to poor spectral efficiency. In order to use the spectrum efficiently overlapping of the sub channels

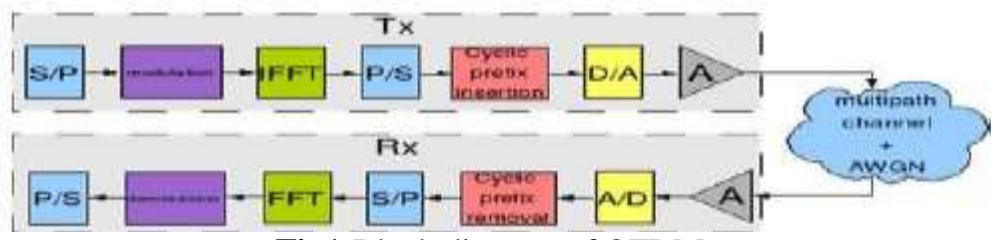

Fig1:Block diagram of OFDM
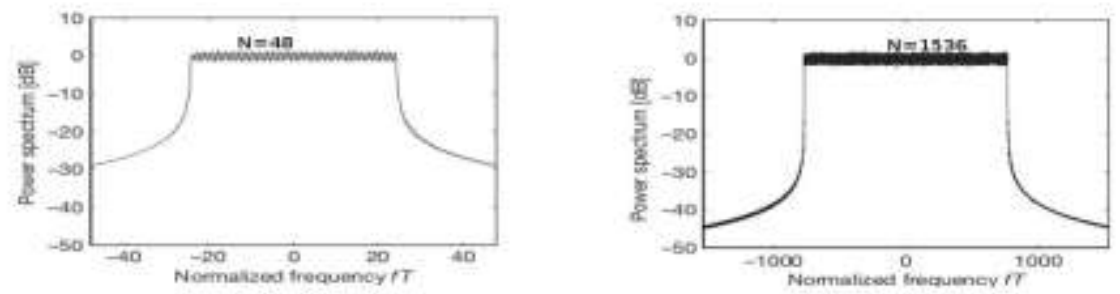

Fig2: OFDM spectrum for $\mathrm{N}$ sub carriers 
is done but problem is that it introduces crosstalk between sub carriers which is eliminated by introducing orthogonal between the sub carriers which is a precise mathematical relationship between the frequencies of the carriers of the system. Frequency selective channels are characterized either by their delay spread or by the channel coherence bandwidth. In a single carrier system a single fade or interference can cause the whole link to fail but in multi carrier systems only a few of the total sub carriers will be affected. In this way multipath fading is eliminated. The main disadvantage of OFDM is the high peak-to-average power ratio (PAPR). When the OFDM is transformed to time domain the resulting signal is the sum of all the sub carriers and when all the subcarriers add up in phase the result is a peak $\mathrm{N}$ times higher than the average power. High PAPR degrades performance of OFDM signals by forcing the analog amplifier to work in nonlinear region, distorting the signal and making the amplifier to consume more power.

In this paper, a clipping method, selective mapping method and partial transmit scheme along with the threshold technique and binary code has been simulated. The methods have been simulated with Matlab and the simulation results show that proposed technique performs better than the existing ones with low computational complexity.

\section{Papr Reduction Techniques}

Peak power occurs when modulated symbols are added with the same phase. So PAPR is the ratio of maximum power to the average power of the signal. It is given as

$$
P A P R=10 \cdot \log _{10} \frac{\operatorname{Max}\left\{\left|x_{n}\right|^{2}\right\}}{E\left[\left|x_{n}\right|^{2}\right]}(d B)
$$

PAPR reduction techniques are two types. They are Distortion based techniques and Redundancy based techniques. In distortion based techniques the time domain signals are directly supressed for which the power signal exceeds a certain threshold level. Some of the examples of this type are clipping method, PTS method etc. In Redundancy based techniques number of candidate signals are generated and then selects the one candidate signal which will have lowest PAPR for actual transmission. Some of the examples of this type are SLM method, Tone reservation method, Tone rejection method, etc.

\section{Clipping Method}

Clipping is one of the most simple and effective methods of PAPR reduction. In this method when the large peak that exceeds a certain threshold level value and occurs infrequently is clipped deliberately. The value of the threshold is chosen in such a way that it provides good PAPR reduction with less BER. Clipping is a nonlinear process and may cause significant in-band distortion that causes BER; out-band distortion causes degradation in spectral efficiency. Let the complex base band of signal is clipped such that the maximum absolute value of $x$ is $A$ that is $|x|=A$, taken absolute of each element of $|x|$ that is $x_{1}, x_{2}, x_{3}, x_{4} \ldots \ldots x_{n}$ and if any $\mathrm{x}$ 's exceeds $\mathrm{A}$, it is clipped so that maximum absolute value of $\mathrm{x}$ 's is $\mathrm{A}$.

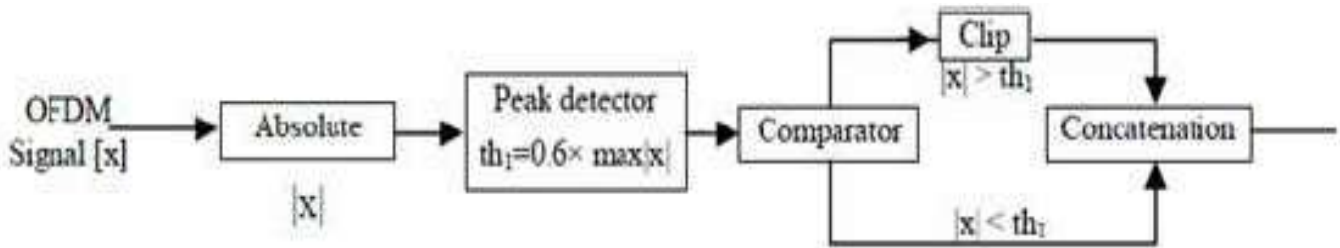

Clipping is accomplished by

$\mathrm{x}=\mathrm{A}$ if $\mathrm{x}>\mathrm{A}$

Fig3: Block diagram of clipping method

$$
x=x \text { if } \quad x \leq A \text { [4] }
$$

After clipping let $\mathrm{x}$ be the received signal (assuming no addition of external noise) but due to clipping, there is certain bit error rate (BER).

$$
\operatorname{PAPR}^{1}=\frac{\max \left\{x^{2}\right\}}{E\left[x^{2}\right]}[5]
$$

\section{Selective Mapping Method}

SLM is a probabilistic technique for PAPR reduction with the aim of reducing the occurrence of peaks in a signal. In this method a set of candidate signals is generated. Before transmitting the signal, its PAPR is calculated and the one with least value of PAPR is transmitted. The actual transmit signal lowest PAPR is selected from a set of sufficiently different signals which all represents the same information. SLM method is very flexible as they do not impose any restriction on modulation applied in the subcarriers or on their number, 
but the only disadvantage is increased computational complexity and increased overheads of side information. Let's data stream after serial to parallel conversion as $\mathrm{X}=\left[\mathrm{X}_{0}, \mathrm{X}_{1, \ldots \ldots \ldots . . .} \mathrm{X}_{\mathrm{n}}\right]^{\mathrm{T}}$.

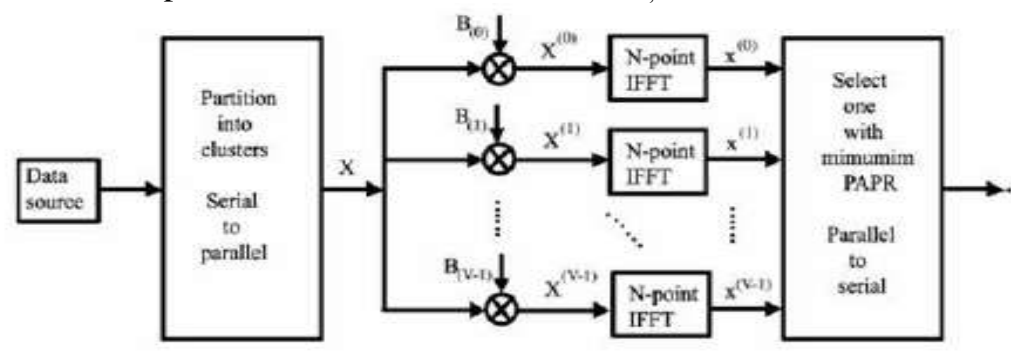

Fig4:Block diagram of SLM Method

Initially each input each $X_{n}^{u}$ can be defined as equation

and $B^{u}$ can be written as

$$
x_{n}^{u}=x_{n} * b_{n}^{u}[6]
$$

$$
x_{n}^{u}=\left[x_{0}^{u}, x_{1}^{u} \ldots \ldots . x_{N-1}^{u}\right][7]
$$

Where $\mathrm{u}=0,1,2 \ldots . \mathrm{U}$ to make the $\mathrm{U}$ phase rotated OFDM data blocks. All $\mathrm{U}$ phased rotated OFDM data blocks represented the same information as the unmodified OFDM data block provided that the phase sequence is known. Output data of the lowest PAPR is selected to transmit. PAPR reduction effect will be better as the copy block $U$ is increased. SLM method effectively reduces PAPR without any signal distortion but it has higher system complexity and computational burden.

\section{Partial Transmit Scheme}

PTS method is a distortion less phase optimization scheme provides reduction of PAPR with a small amount of redundancy. The input data is divided in to number of disjoint sub blocks and they are weighted by a set of phase factors to create a set of candidate signals. Finally the candidate signal with the lowest PAPR with the help of threshold is chosen for transmission.

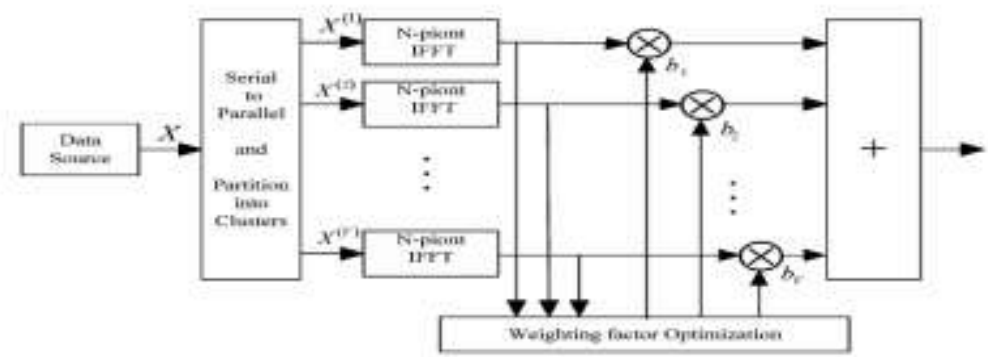

Fig5: Block diagram of Partial Transmit Scheme

Let $\mathrm{N}$ denote the number of subcarriers used for parallel information transmission and $\mathrm{x}_{\mathrm{k}}(0 \leq \mathrm{k} \leq \mathrm{N}-1)$ represent the $\mathrm{k}^{\text {th }}$ complex modulated symbol in a block of information symbols. As shown in the figure for PAPR reduction using PTS scheme, the frequency domain vector $\mathrm{X}$ is partitioned into disjoint $\mathrm{V}$ sub blocks, which are represented by $X^{v}, v=1,2,3 \ldots V$. Hence

$$
X=\sum_{v=1}^{V} X^{v}[8]
$$

where $\mathrm{X}^{\mathrm{v}}=\left[X_{0}^{v}, X_{1}^{v}, X_{2}^{v} \ldots \ldots X_{N-1}^{v}\right]$ with $X_{k}^{v}=X_{k}$ or $0(1 \leq \mathrm{v} \leq \mathrm{V})$. let $\mathrm{b}=\mathrm{b}_{\mathrm{v}}=\exp (\mathrm{j} \theta v), \mathrm{v}=1,2,3 \ldots \mathrm{V}$ be the set of phase factors which are applied to the sub blocks $X^{v}$. The substitute frequency domain signals are

$$
\mathrm{X}^{\mathrm{l}}=\sum_{v=1}^{V} b_{v} X^{v},\left(b_{v}=\exp (j \theta v)\right)[9]
$$

Note that these partial sequences are independently rotated by phase factors $b$, taking the IDFT of the above equation and using the linearity property of the IDFT, the time domain partial transmit sequences can be expressed as

$$
\mathrm{x}^{\mid}=\operatorname{IDFT}\left(\mathrm{X}^{\mid}\right)=\sum_{v=1}^{V} b_{v} \operatorname{IDFT}\left(X^{v}\right)=\sum_{v=1}^{V} b_{v} x^{v}[10]
$$

The objective is to optimally combine the $\mathrm{V}$ sub blocks to obtain the time domain OFDM signals with the lowest PAPR. Without any loss of performance, one can set $b_{1}=1$ and observe that there are (V-1) sub blocks to be optimized. Consequently, to achieve the optimal phase factor for each input data sequence (assume that there are $\mathrm{W}$ phase vectors in the phase set), $\mathrm{W}^{\mathrm{v}-1}$ combinations should be checked in order to obtain the minimum PAPR. Therefore, the search complexity for an optimum set of the phase factors increases exponentially with the number of sub blocks. 


\section{Proposed Pts Scheme With Low Complexity}

A PTS scheme is presented based on listing the phase factors into multiple subsets table and utilizing the correlation among the phase factors in each subset, in order to reduce the computational complexity. Here, we firstly introduce the concept of 'basis vector of the phase factors': for a PTS based OFDM system with V=2 and $\mathrm{W}=2$, we can list all phase factors: $\mathrm{b}_{1}=[1,1], \mathrm{b}_{2}=[1,-1]$. For this reason, we can take $\mathrm{B}_{1}=[1,1]$ as the basis vector of the phase factors, provided the sign of the elements is not considered. As for $\mathrm{V}=2$ and $\mathrm{W}=4$, all phase factors are $b_{1}=[1,1], b_{2}=[1,-1], b_{3}=[1, j], b_{4}=[1,-j]$, so the basis vectors of the phase factors can be written as $\mathrm{B}_{1}=[1,1], \mathrm{B}_{2}=[1, \mathrm{j}]$ (see as a real number).

Then, list all phase weighting vectors in the table subject to the following rules:

i. Find the basis vectors of all phase weighting vectors and put them in the first row, note that only one element in the adjacent basis vectors is different.

ii. In each column, the phase weighting vectors have the same basis vector.

iii. For the adjacent phase weighting vectors in the same column, only the sign of one element is different.

iv. The sign of the last phase weighting vectors in one column is the same as the first weighting vectors in the next column.

\section{Performance Analysis}

By employing the threshold to the PAPR reduction techniques the computational complexity is reduced. The computational complexity of a PTS based can also be reduced by reducing the number of candidate signals, but the computational complexity is reduced at the cost of performance loss for PAPR reduction. Unlike these methods without the loss of performance PAPR can reduced based on the threshold so that if the required PAPR is threshold is fixed, then the candidate signal of the lowest PAPR is only transmitted. The sub block partition method that is used is interleaved partition.

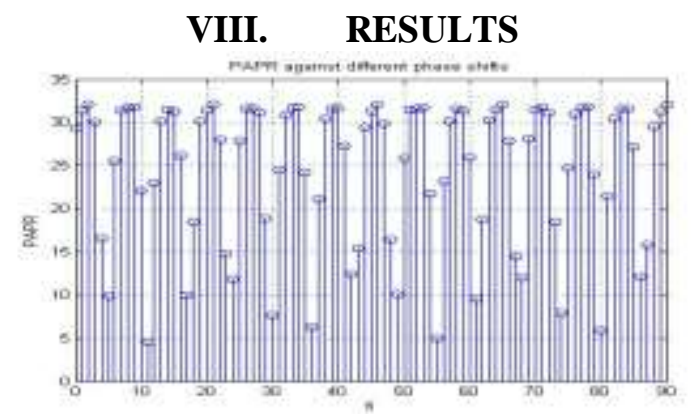

Fig6: SLM Method

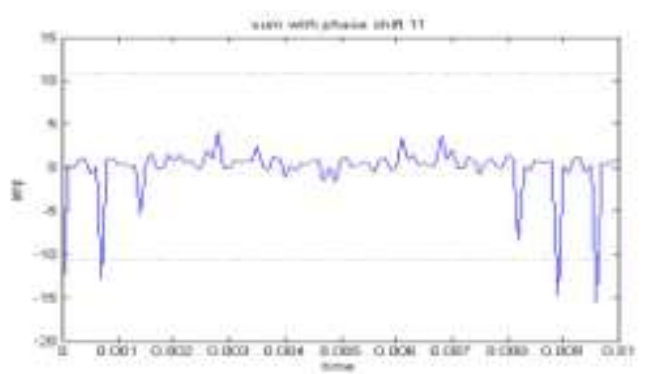

Fig7: SLM method when Phase shift is 11

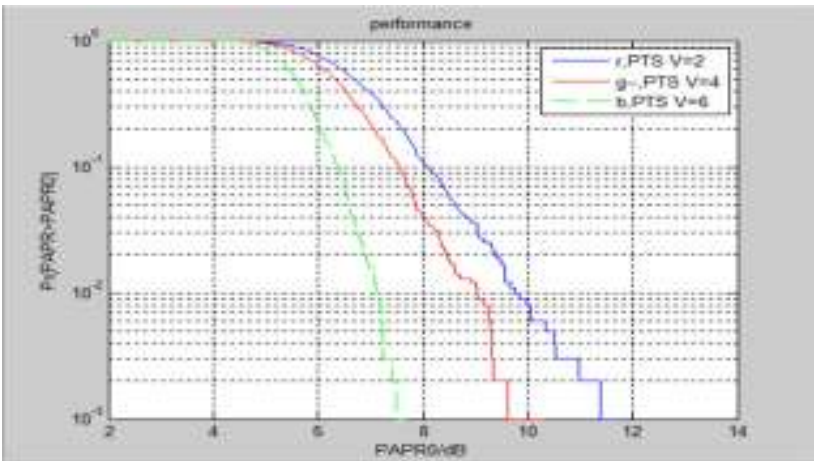

Fig8: Basic PAPR in the OFDM systems 


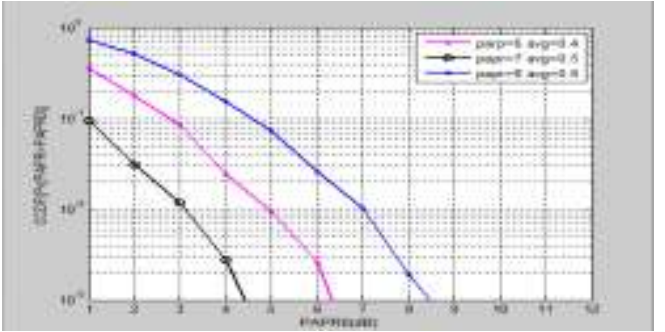

Fig9: Reduced PAPR using Clipping Method

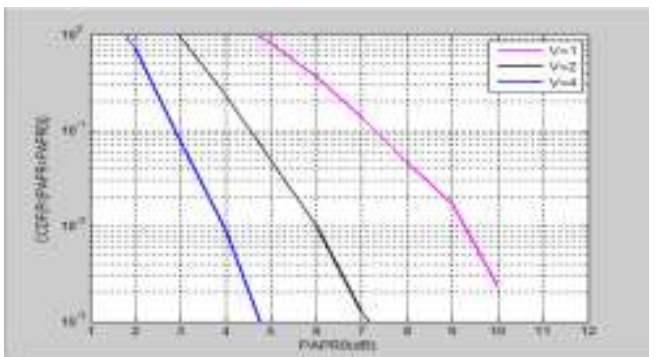

Figure10: $\underline{\text { Reduced PAPR using PTS scheme with threshold }}$

\section{Conclusion\& Forthcomings}

High PAPR of transmitted signal is one of the major drawbacks of OFDM systems. In the PTS scheme, the computational complexity that has been increased extensively with the number of sub blocks, in order to reduce this complexity has been reduced by utilizing the correlation among the candidate signals. In addition to that with the addition of Threshold technique, the PAPR value has been reduced. On comparison with the Clipping method and SLM method the proposed method has given the best PAPR results required.In the OFDM systems the orthogonality can be obtained using the Walsh Hadmard codes, IIFT instead of IDFT.

\section{Acknowledgement}

I would like to express sincere thanks to our project guide Mr.Faruk umar shaik[Ph.d], M.Tech,The HOD, Department of Electronics and communication Engineering, Quba college of Engineering, Nellore. I would like to express my thanks to Mr.P.Praveen, M.Tech, The Engineer at Qualcomm, Hyderabad who gave me the ideas to the project. Finally I would like to thank all teaching, non-teaching staff, colleagues and my parents for their sincere help to complete my project without any problem.

\section{References}

[1] Xue Li,Vasu.D.Chakravarthy "Spreading Code Design of Adaptive Non-Contiguous SOFDM for Dynamic Spectrum Access", IEEE Journal of Selected Topics in Signal Processing,vol.5,no.1,Jan. 2011.

[2] Henrik Schulze and Christian Luders. "Theory and Applications of OFDM and CDMA. Wideband Wireless Communications". Wiley, 2003.

[3] Y.Wu and W. Y. Zou, "Orthogonal frequency division multiplexing: A multi-carrier modulation scheme," IEEE Trans. Consumer Electronics, vol. 41, no. 3, pp. 392-399, Aug. 1995.

[4] N. Dinur and D. Wulich, "Peak-to-average power ratio in high-order OFDM," IEEE Trans. Communications, vol. 49, no. 6, pp. 10631072, Jun. 2001.

[5] Lingyin Wang and Ju Liu, Senio "PAPR Reduction of OFDM Signals by PTS With Grouping and Recursive Phase Weighting Methods" IEEE Transactions on Broadcasting,vol.43,no.1,Jan. 2010.

[6] Jun Hou, Jianhua Ge, and Jing Li "Peak-to-Average Power Ratio Reduction of OFDM Signals Using PTS Scheme With Low Computational Complexity" IEEE Transactions on Broadcasting, vol.57, no.1, March 2011

[7] Ms. V. B. Malode, Dr. B. P. Patil "PAPR Reduction Using Modified Selective Mapping Technique".Int. J. of Advanced Networking and Applications, Volume:02, Issue: 02, Pages:626-630 (2010).

[8] G.Yue, X.Wang,M.Madihian "Peak-to-Average Power Ratio Reduction scheme with Threshold limited selection for orthogonal frequency division multiplexing" IET Communications Vol. 2, No. 8, pp. 1034-1042,2008.

[9] Heung-Gyoon Ryu, Member, IEEE, Jae-Eun Lee and Jin-Soo Park, Member, IEEE "Dummy Sequence Insertion (DSI) for PAPR Reduction in the OFDM Communication System" IEEE Transactions on Consumer Electronics, Vol. 94 50, No. 1, FEBRUARY 2004.

[10] Y. Zhou and T. Jiang, "A novelclipping integrated into ACE for PAPR reduction in OFDM systems," 2009 International Conference on Wireless Communications and Signal Processing, WCSP 2009, Mar.2009.

\section{Biographies}

Ms.Nilofer.SKhas Received B.tech in Electronics and Communication Engineering (ECE) from JNTU, Anantapur, India i 2009. She is persuing her M.Tech in Quba college of engineering and technology, JNTUA, Anantapur.

Mr. Shaik. Umar Faruq is working as an Associate Professor in QUBA College of engineering and Technology, Nellore. He has 9 years teaching experience both at UG and PG level and 1 year industrial experience. 